\title{
Targeting transcription of MCL-1 sensitizes HER2- amplified breast cancers to HER2 inhibitors
}

\author{
Konstantinos V. Floros ${ }^{1}$, Sheeba Jacob', Richard Kurupi ${ }^{1}$, Carter K. Fairchild ${ }^{1}$, Bin Hu², Madhavi Puchalapalli², \\ Jennifer E. Koblinski ${ }^{2}$, Mikhail G. Dozmorov' ${ }^{3}$, Sosipatros A. Boikos ${ }^{4}$, Maurizio Scaltriti $\mathbb{B}^{5,6,7}$ and Anthony C. Faber (1)
}

\begin{abstract}
Human epidermal growth factor receptor 2 gene (HER2) is focally amplified in approximately 20\% of breast cancers. HER2 inhibitors alone are not effective, and sensitizing agents will be necessary to move away from a reliance on heavily toxic chemotherapeutics. We recently demonstrated that the efficacy of HER2 inhibitors is mitigated by uniformly low levels of the myeloid cell leukemia 1 (MCL-1) endogenous inhibitor, NOXA. Emerging clinical data have demonstrated that clinically advanced cyclin-dependent kinase (CDK) inhibitors are effective MCL-1 inhibitors in patients, and, importantly, well tolerated. We, therefore, tested whether the CDK inhibitor, dinaciclib, could block MCL1 in preclinical HER2-amplified breast cancer models and therefore sensitize these cancers to dual HER2/EGFR inhibitors neratinib and lapatinib, as well as to the novel selective HER2 inhibitor tucatinib. Indeed, we found dinaciclib suppresses MCL-1 RNA and is highly effective at sensitizing HER2 inhibitors both in vitro and in vivo. This combination was tolerable in vivo. Mechanistically, liberating the effector BCL-2 protein, BAK, from MCL-1 results in robust apoptosis. Thus, clinically advanced CDK inhibitors may effectively combine with HER2 inhibitors and present a chemotherapyfree therapeutic strategy in HER2-amplified breast cancer, which can be tested immediately in the clinic.
\end{abstract}

\section{Introduction}

HER2 inhibitors extend survival in HER2-amplified breast cancers; however, they are not sufficiently active as monotherapy ${ }^{1,2}$, unlike other receptor tyrosine kinase (RTK) inhibitors in solid tumor cancer paradigms. Due to this, there remains a reliance on chemotherapy; in contrast, in paradigms like epidermal growth factor receptor (EGFR)-mutant lung cancer and anaplastic lymphoma kinase $(A L K)$-translocated lung cancer, effective targeted therapy has mitigated the need of chemotherapy ${ }^{3}$.

Correspondence: Anthony C. Faber (acfaber@vcu.edu)

${ }^{1}$ Department of Oral and Craniofacial Molecular Biology, Philips Institute for Oral Health Research, VCU School of Dentistry and Massey Cancer Center, Virginia Commonwealth University, Richmond, VA 23298, USA

2Department of Pathology, Virginia Commonwealth University School of

Medicine and Massey Cancer Center, Richmond, VA 23220, USA

Full list of author information is available at the end of the article

These authors contributed equally: Konstantinos V. Floros, Sheeba Jacob,

Richard Kurupi

Edited by L. Galluzzi
We have demonstrated recently that HER2-amplified breast cancers have significantly lower NOXA levels, leading to MCL-1-mediated resistance to HER2 inhibitors through suppression of apoptosis ${ }^{4}$. Similarly, Merino et al. $^{5}$ demonstrated that co-administration of MCL-1 inhibitors with HER2 inhibitors sensitizes HER2-amplified breast cancer models. While MCL-1 BH3 mimetics are advancing into clinical trials either alone or with venetoclax in hematological cancers, it remains uncertain whether these drugs will be able to sufficiently block the interaction of MCL-1 and proapoptotic BH3-only proteins such as NOXA and BIM. Moreover, the tolerability of these drugs in combination is unkown.

Inhibitors that block CDK9 can interfere with gene transcription. Thus, transcription of mRNAs with short half-lives that need to be synthesized at a high rate may be particularly affected by these agents ${ }^{6}$. Unique among the antiapoptotic proteins, MCL-1 has a very short half-life ${ }^{7,8}$. Dinaciclib has been used as an MCL-1 inhibitor in several cancer paradigms. It has already been reported that

\section{(c) The Author(s) 2021}

(c) (i) Open Access This article is licensed under a Creative Commons Attribution 4.0 International License, which permits use, sharing, adaptation, distribution and reproduction cc) in any medium or format, as long as you give appropriate credit to the original author(s) and the source, provide a link to the Creative Commons license, and indicate if changes were made. The images or other third party material in this article are included in the article's Creative Commons license, unless indicated otherwise in a credit line to the material. If material is not included in the article's Creative Commons license and your intended use is not permitted by statutory regulation or exceeds the permitted use, you will need to obtain permission directly from the copyright holder. To view a copy of this license, visit http://creativecommons.org/licenses/by/4.0/. 
dinaciclib causes mitochondria-dependent apoptosis in osteosarcoma with MCL-1 being the primary target ${ }^{9}$, and in hepatocellular carcinoma dinaciclib decreases $M C L-1$ mRNA levels without significantly changing the expression of other BCL-2 proteins ${ }^{10}$. Interestingly, CDK9 inhibition with dinaciclib is highly effective in MYCdriven lymphomas and involves downregulation of MCL- $1^{11}$. And while there are also studies that support the elimination of MCL-1 at the protein level as the potential mechanism of action of dinaciclib ${ }^{12}$, most advocate for transcriptional downregulation of $M C L-1$ as the critical mechanism ${ }^{9,13}$. In addition, we have recently demonstrated that the CDK inhibitor dinaciclib effectively blocks MCL-1 to sensitize EGFR inhibitors in EGFRmutant non-small cell lung cancer (NSCLC) ${ }^{14}$. Dinaciclib exposure time peaks are roughly $2 \mathrm{~h}$ in humans, which is sufficient to block MCL-1, but not sufficient to block CDK1 or $\mathrm{CDK} 2{ }^{15}$. This suggests that the anticancer activity seen with dinaciclib is a result of its inhibitory effect on CDK9, and not CDK1/2. In a phase I trial in breast cancer patients, neutropenia and leukopenia were common, but dinaciclib in general was well tolerated ${ }^{16}$. In this study, we aimed to explore whether dinaciclib was sufficient to sensitize preclinical models of HER2-amplified breast cancer through downregulation of MCL-1.

\section{Results}

Dinaciclib sensitizes HER2-amplified breast cancers to HER2 inhibitors and is superior to the MCL-1 BH3 mimetic A-1210477

We and others recently demonstrated that pharmacological inhibitors of MCL-1 sensitized HER2 inhibitors in $H E R 2$-amplified breast cancers ${ }^{4,5}$. Based both on dinaciclib's ability to inhibit MCL-1 in vitro and in vivo and its intrinsic therapeutic window, we investigated whether dinaciclib could be added to HER2 inhibitors and sensitize them through downregulation of MCL-1. In both HER2amplified BT-474 and MDA-MB-453 cells, dinaciclib effectively reduced MCL-1 expression (Fig. 1A). In both cell lines, dinaciclib was more potent as a combining partner with the HER2 inhibitor lapatinib than was the MCL-1 BH3 mimetic A-1210477, as evidenced by cleaved PARP levels, a marker for apoptosis (Fig. 1A). In addition, while phosphorylation of HER2 was completely abolished, consistent with the on-target effect of lapatinib, HER2 levels were not significantly altered with any of the drug treatments (Fig. 1A). As expected, both the HER2/PI3K/ TORC1 and HER2/RAS/TORC1 signaling pathways were disrupted by HER2 kinase inhibition, as evidenced by loss of pHER2, p-AKT (PI3K readout), p-ERK (RAS pathway readout), and $\mathrm{p}-\mathrm{S} 6$ loss (mTORC1 pathway readout $)^{17}$ (Fig. 1A). Dinaciclib strongly activated PI3K and MEK signaling, as evidenced by increased p-AKT (308) and pERK, respectively. However, lapatinib eventually abrogated both feedback activations (Fig. 1A). Of note, downregulation of MCL-1 by dinaciclib destabilizes also BIM EL (Fig. 1A), which was also noticed in our previous studies ${ }^{4}$.

In order to corroborate previous reports that dinaciclibinduced MCL-1 decreases are due to loss of MCL-1 transcription $^{10}$, we evaluated $M C L-1$ mRNA expression after treating different $H E R 2$-amplified breast cancer cell lines with dinaciclib (Fig. 1B). As expected, $M C L-1$ mRNA expression was suppressed $2 \mathrm{~h}$ after dinaciclib addition. Consistently, after treating BT-474 cells for $24 \mathrm{~h}$ and the less sensitive MDA-MB- 453 cells for $72 \mathrm{~h}$, cell viability decreased more with the combination of lapatinib and dinaciclib than with lapatinib and A-1210477 (Fig. 1C). We further determined the sensitivity of the HER2amplified breast cancer cell lines to the different combinations of these agents to gain information regarding the contribution of each single agent to the observed toxicity (Supplementary Fig. 1). In line with our previous data, dinaciclib displays a more synergistic potential with lapatinib than A-1210477 does. Altogether, these data indicate that dinaciclib downregulates MCL-1 and sensitizes to HER2 inhibitor in HER2-amplified breast cancers. Given that PARP cleavage has been reported to be implicated in other non-apoptotic processes ${ }^{18,19}$ and MCL-1 also exhibits apoptosis-independent functions in the cell ${ }^{20,21}$, we assessed Annexin V positivity by flow cytometry to confirm toxicity from loss of MCL-1 was due to an increase in apoptosis (Fig. 1D and Supplementary Fig. 2). To gain mechanistic insight, we immunoprecipitated MCL-1 in the BT-474 and MDA-MB-453 cells and observed that dinaciclib toxicity is mediated at least in part by BAK, which is liberated from MCL-1 following treatment and is free to execute its apoptotic program (Fig. 1E). Potential alterations in BIM EL:MCL-1 complexes were also investigated since BIM EL is a direct activator of $\mathrm{Bcl}-2$-associated $\mathrm{X}$ protein $(\mathrm{BAX}) / \mathrm{Bcl}-2$ homologous antagonist/killer (BAK) molecules and its liberation could lead to further cell death responses. However, consistent with our previous data ${ }^{22}$, BIM EL levels were significantly downregulated in the whole-cell lysates following the addition of dinaciclib (Fig. 1E) making likely its role in combination toxicity, if any, limited.

\section{Dinaciclib sensitization to HER2-amplified breast cancers is abrogated by BAK knockdown and largely mediated by MCL-1}

As BAK-MCL-1 was sharply disrupted by dinaciclib, we sought to investigate this complex further and the role, if any, of BAK in dinaciclib and HER2 inhibitor/dinaciclib toxicity. Mechanistically, MCL-1 binds to BAK to prevent its activation ${ }^{23}$. Thus, if MCL-1 is critical to combination activity, BAK knockdown should mitigate the activity of 


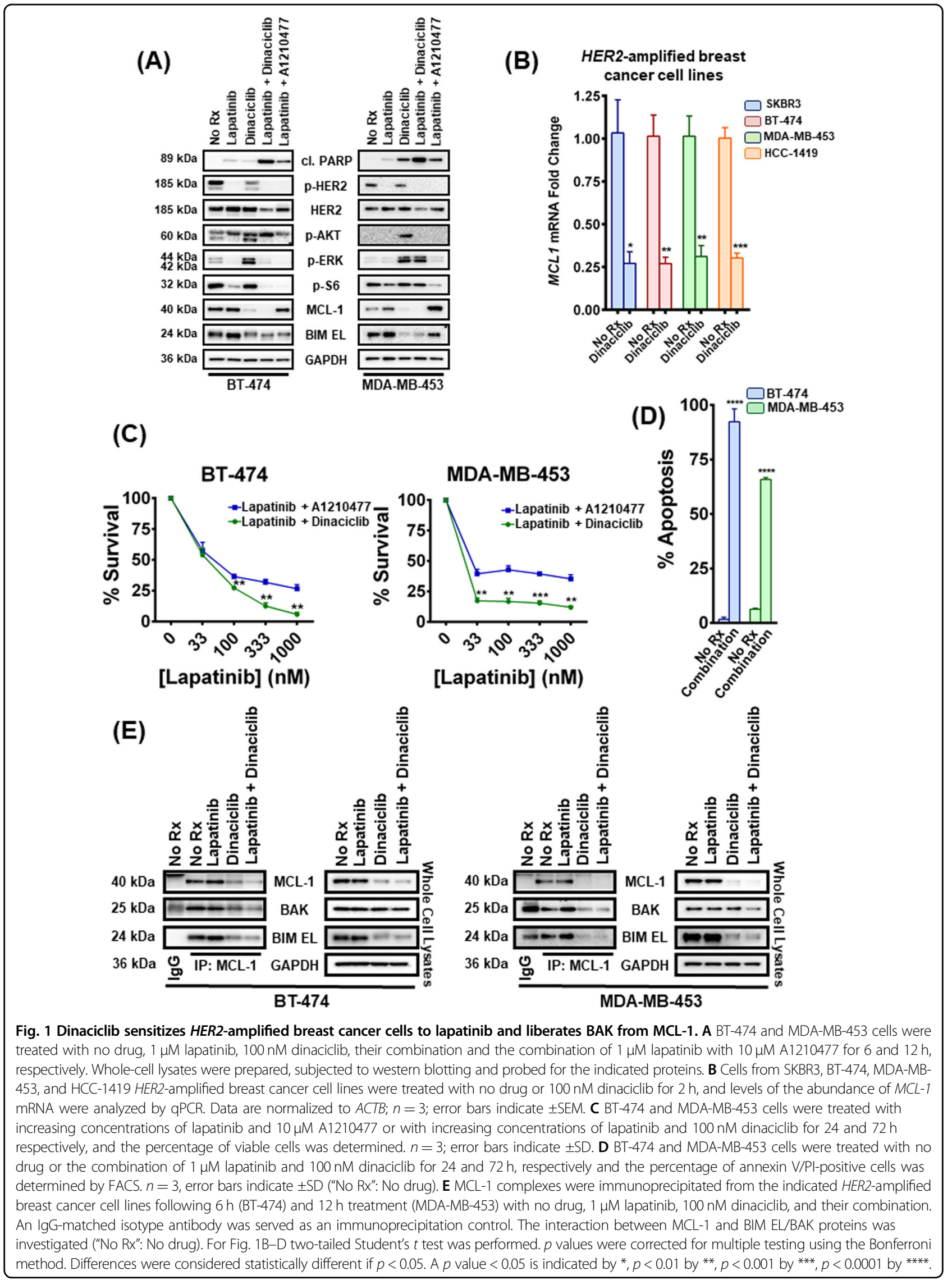



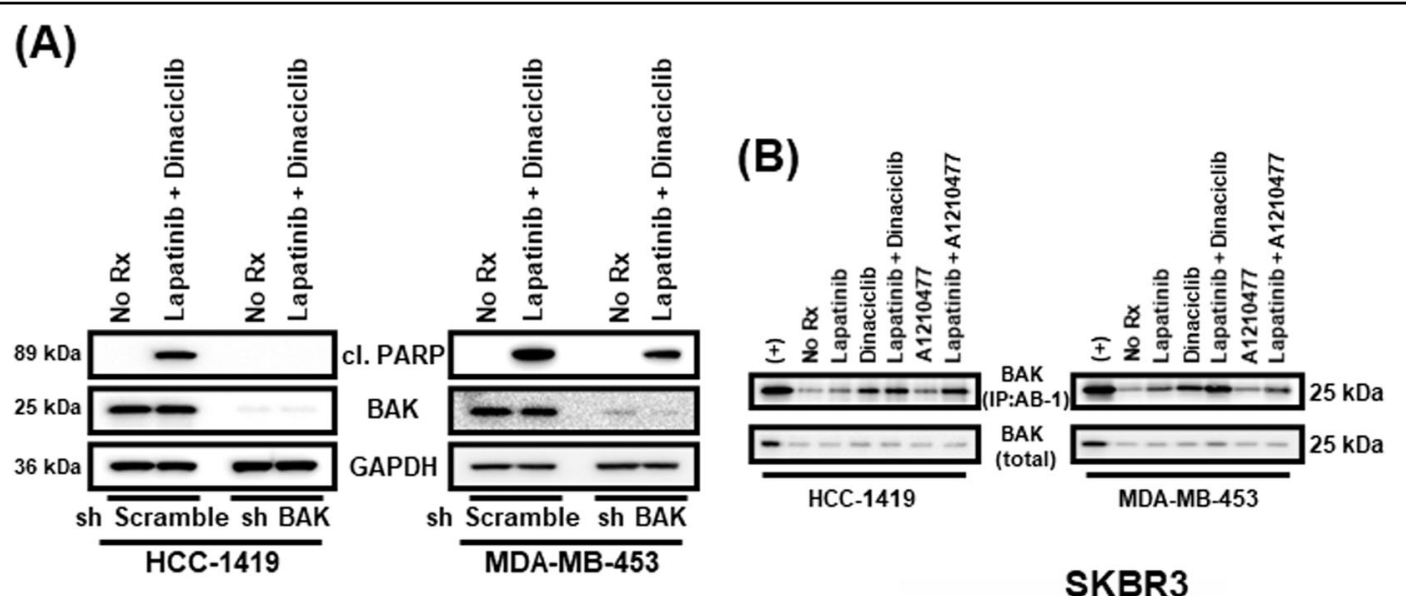

(C)
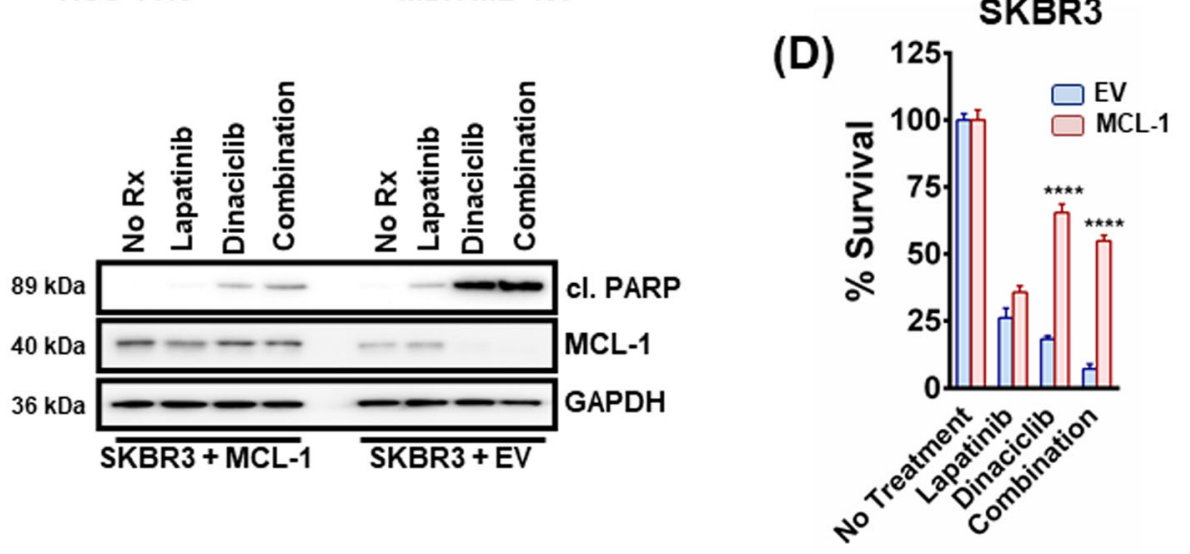

(E)
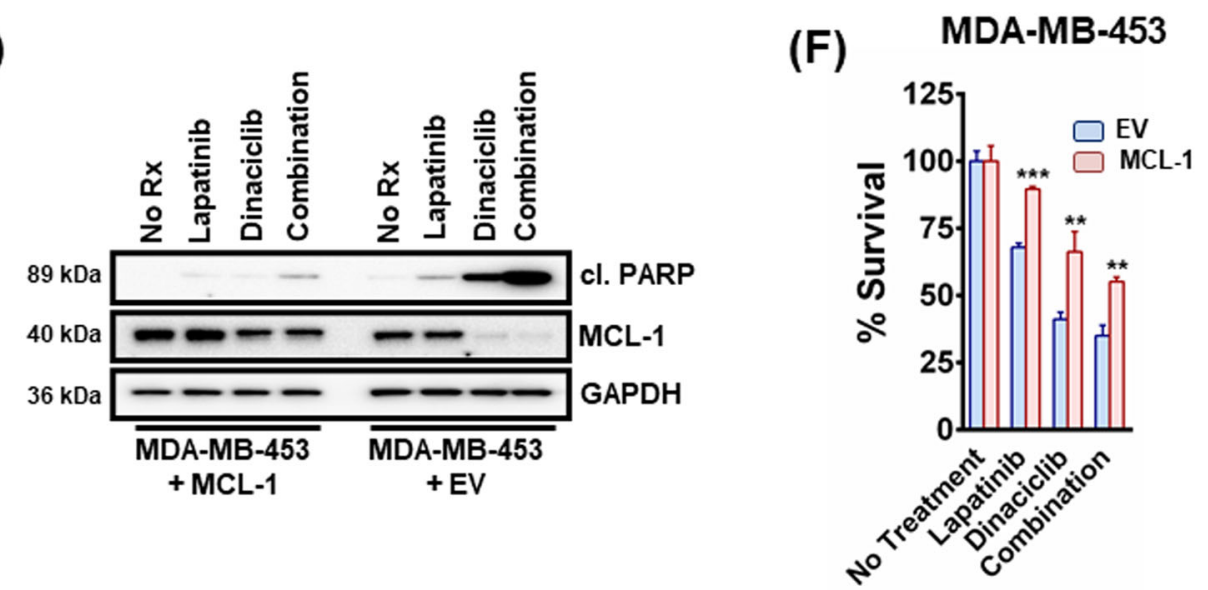

Fig. 2 (See legend on next page.)

the combination of dinaciclib and HER2 inhibition. Indeed, we found reduction of BAK by shRNA led to loss of apoptotic activity of the combination in two HER2amplified HCC-1419 and MDA-MB-453 breast cancer cell lines where we were able to achieve sufficient knockdown (Fig. 2A). We next immunoprecipitated BAK with an antibody that exploits a conformation change in BAK upon its activation and only recognizes this active BAK species $^{24}$. Consistent with an important role of MCL-1: BAK in combination toxicity, BAK was activated following either dinaciclib or A1210477 exposure, which was exacerbated upon the addition of lapatinib in both cases (Fig. 2B). Consistent with the enhanced apoptotic activity of the dinaciclib/lapatinib combination (Fig. 1A, B), BAK 
(see figure on previous page)

Fig. 2 BAK is required for dinaciclib-induced cell death in HER2-amplified breast cancer cells and dinaciclib functions mainly by inhibiting

MCL-1. A HCC-1419 and MDA-MB-453 cells were transduced with lentiviruses containing plasmids with an shRNA sequence targeting BAK or a nontargeting control. Puromycin-resistant cells were pooled after each infection. Cells were then treated with no drug, $1 \mu \mathrm{M}$ lapatinib, $100 \mathrm{nM}$ dinaciclib or their combination overnight. Cell lysates were prepared and subjected to western blotting and probed for cleaved PARP, BAK, and GAPDH ("No

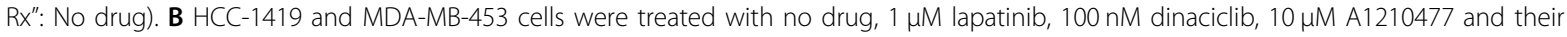
combinations (lapatinib/dinaciclib and lapatinib/A1210477) overnight and CHAPS lysates (using the zwitterionic detergent CHAPS, that can solubilize cells without promoting significant conformational changes in BAX and BAK, including the $\mathrm{N}$-terminal Bak epitope exposure recognized by antibody Ab-1) were prepared and subjected to AB-1 IP and western blotting. Total cell lysates were analyzed in parallel. C SKBR3 control or MCL-1-expressing cells were treated with $1 \mu \mathrm{M}$ lapatinib, $100 \mathrm{nM}$ dinaciclib, and their combination for $12 \mathrm{~h}$. Whole-cell lysates were prepared, subjected to western blotting and probed for the indicated proteins. D SKBR3 control or MCL-1-expressing cells were treated with $1 \mu \mathrm{M}$ lapatinib, $100 \mathrm{nM}$ dinaciclib, and their combination for $12 \mathrm{~h}$ and subjected to CellTiter-Glo. $n=3$; error bars indicate \pm SD. E MDA-MB-453 control or MCL-1-expressing cells were treated with $1 \mu \mathrm{M}$ lapatinib, $100 \mathrm{nM}$ dinaciclib, and their combination for $12 \mathrm{~h}$. Whole-cell lysates were prepared, subjected to western blotting and probed for the indicated proteins. F MDA-MB-453 control or MCL-1-expressing cells were treated with $1 \mu \mathrm{M}$ lapatinib, $100 \mathrm{nM}$ dinaciclib and their combination for $72 \mathrm{~h}$ and subjected to CellTiter-Glo. $n=3$; error bars indicate \pm SD. For Fig. 2D, F two-tailed Student's $t$ test was performed. $p$ values were corrected for multiple testing using the Bonferroni method. Differences were considered statistically different if $p<0.05$. A $p$ value $<0.05$ is indicated by ${ }^{*}, p<0.01$ by **, $p<0.001$ by **, and $p<0.0001$ by ***. EV: empty vector, (+): positive control, CHAPS: 3- ((3-cholamidopropyl) dimethylammonio)-1-propanesulfonic acid.

was more active following dinaciclib/lapatinib than A1210477/lapatinib therapy (Fig. 2B).

While these data demonstrated a role of the MCL1-BAK complex in dinaciclib/HER2 inhibitor combination efficacy, we sought to investigate how important the MCL-1-BAK complex was to combination efficacy. For these experiments, in addition to the MDA-MB-453 cells, we used the SKBR3 HER2-amplified breast cancer cell line, which is very sensitive to MCL-1 inhibition ${ }^{4,25}$. We found that the expression of exogenous MCL-1 was sufficient to mitigate the efficacy of both single-agent dinaciclib and the combination of dinaciclib and lapatinib to induce cell death (Fig. 2C), which translated into increased viability (Fig. 2D). In the MDA-MB-453 cells, rescue of MCL-1 expression was sufficient to block cell death (Fig. 2E) and increase total cell viability (Fig. 2F). To investigate if the other main pro-survival BCL2 proteins are implicated in dinaciclib-mediated apoptosis, we transiently overexpressed BCL2 and BCL-xL in the same two cell lines and treated with lapatinib, dinaciclib, and their combination (Suplementary Fig. 3 and Suplementary Fig. 4). Increased levels of BCL2 as well as BCL-xL did not result in significant suppression of the toxicity caused by the single agents or their combination, as determined by cleaved PARP expression (Suplementary Fig. 3A, C) or cell viability measurement (Suplementary Fig. 3B, D), demonstrating an MCL-1-specific effect caused by dinaciclib. However, while we did not see a sensitizing effect of the BCL-2 inhibitor venetoclax to lapatinib in the HER2amplified breast cancer cell lines BT-474 or MDA-MB453, we did see added toxicity with the tool BCL-xL inhibitor A-1331852, which was similar to that afforded by A-1210477 (Suplementary Fig. 4A, C). Similarly, A1331852 sensitized the BT-474 and MDA-MB-453 cells to dinaciclib while venetoclax either did not (BT-474) or had a minimal effect (MDA-MB-453); strikingly, however,
A-1210477 had no sensitizing effect on dinaciclib, consistent with MCL-1 as the key dinaciclib target in HER2amplified breast cancer (Suplementary Fig. 4B, D).

\section{Dinaciclib sensitizes HER2-amplified breast cancer cells to the novel, selective HER2 inhibitor tucatinib}

As there are now at least seven FDA-approved HER2 inhibitors $^{26}$, we wanted to corroborate our findings with some of the newer HER2 inhibitors. Tucatinib is a novel, FDA-approved agent that has demonstrated more than 1000-fold selectivity for HER2 over EGFR in in vitro assays $^{27}$ and significant efficacy in clinical trials for the treatment of metastatic HER2-positive breast cancer $(\mathrm{NCT} 02614794)^{28-32}$. As expected from a HER2 inhibitor, tucatinib inhibited p-HER2, p-AKT and p-ERK in the HER2-amplified breast cancer cells BT-474 and MDAMB- $453^{17}$ (Fig. 3A). Addition of dinaciclib sensitizes the cancer cells to tucatinib as evidenced by increased cleaved PARP (Fig. 3A) and decreased cell viability in both cell lines (Fig. 3B, C), with their sensitivity reaching a plateau at about $1000 \mathrm{nM}$ of tucatinib. To verify that complexes of MCL-1 with pro-apoptotic BCL2 proteins were disrupted by dinaciclib, we immunoprecipitated MCL-1 complexes in lysates derived from the MDA-MB-453 cells, following treatment with tucatinib, dinaciclib and their combination (Fig. 3D). Immunoprecipitation complex investigation confirmed that MCL-1:BAK complexes were disrupted following treatment with $100 \mathrm{nM}$ dinaciclib (Fig. 3D).

\section{Dinaciclib is effective in vivo at sensitizing HER2-amplified breast cancers to HER2 inhibitors}

We next determined whether the combination of dinaciclib and lapatinib would be effective in vivo. As mentioned, exposure time, at least in humans, is sufficiently different and prevents the ability of dinaciclib to potently inhibit some CDK targets ${ }^{15}$. We found that 


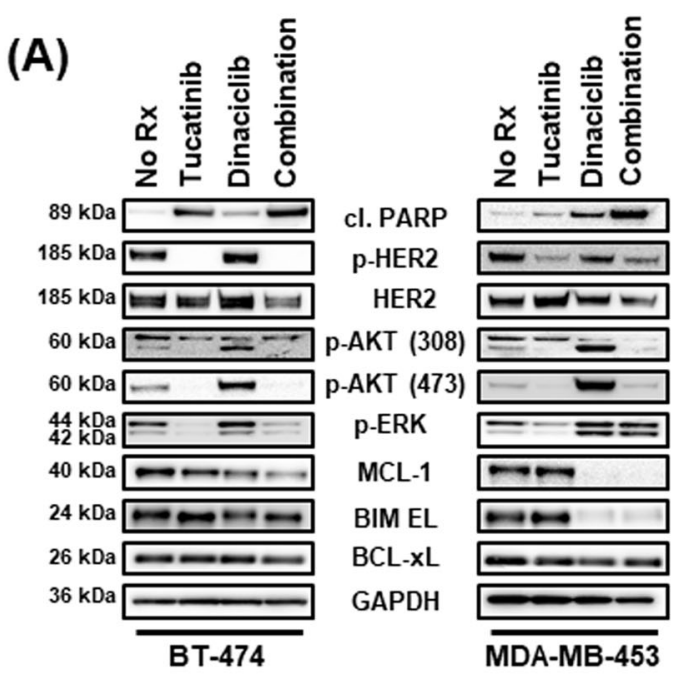

(B)

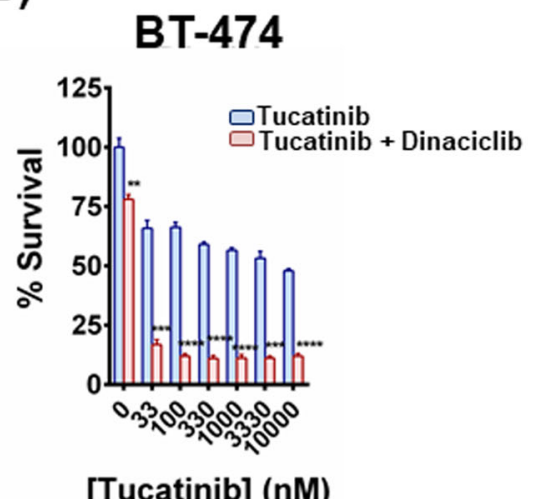

(C)

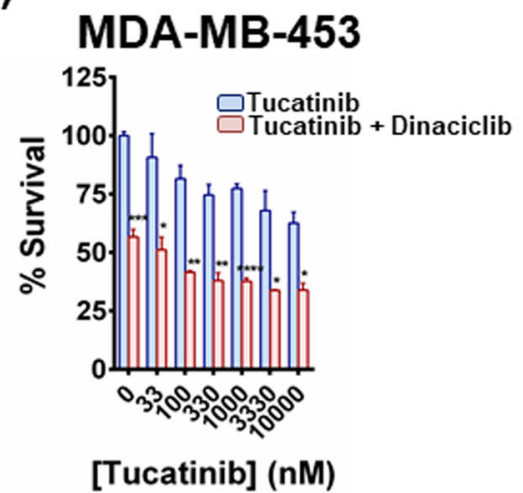

(D)

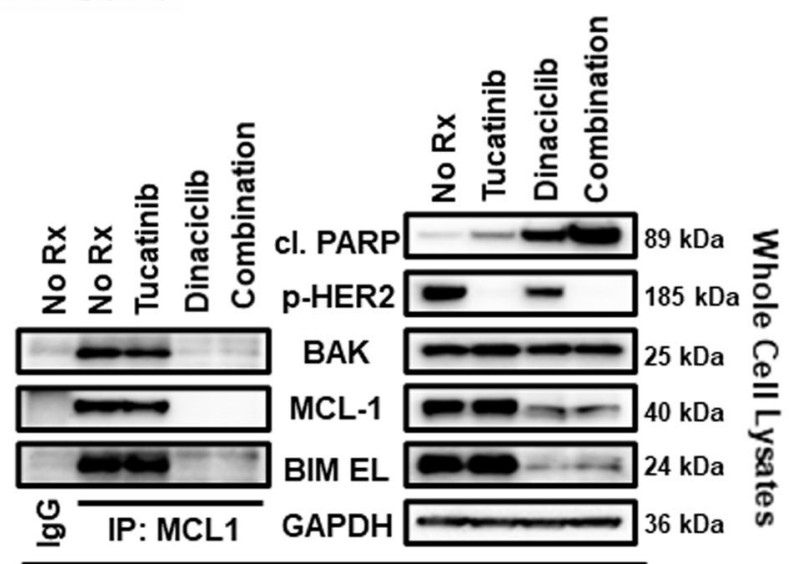

\section{MDA-MB-453}

Fig. 3 Dinaciclib sensitizes HER2-amplified breast cancer cells to tucatinib and liberates BAK from MCL-1. A BT-474 and MDA-MB-453 cells were treated with no drug, $1 \mu \mathrm{M}$ tucatinib, $100 \mathrm{nM}$ dinaciclib, and their combination for 6 and $12 \mathrm{~h}$, respectively. Whole-cell lysates were prepared, subjected to western blotting and probed for the indicated proteins. B BT-474 cells were treated with increasing concentrations of tucatinib and $100 \mathrm{nM}$ dinaciclib for $24 \mathrm{~h}$ and the percentage of viable cells was determined. $n=3$; error bars indicate \pm SD. C MDA-MB-453 cells were treated with increasing concentrations of tucatinib and $100 \mathrm{nM}$ dinaciclib for $48 \mathrm{~h}$ and the percentage of viable cells was determined. $n=3$; error bars indicate \pm SD. D MCL-1 complexes were immunoprecipitated from MDA-MB-453 cells following $12 \mathrm{~h}$ treatment with no drug, $1 \mu \mathrm{M}$ tucatinib, $100 \mathrm{nM}$ dinaciclib, and their combination. An IgG-matched isotype antibody was served as an immunoprecipitation control. The interaction between MCL-1 and BIM EL/BAK proteins was investigated. For Fig. 3B, C two-tailed Student's $t$ test was performed; $p$ values were corrected for multiple testing using the Bonferroni method. Differences were considered statistically different if $p<0.05$. A $p$ value $<0.05$ is indicated by $*, p<0.01$ by **, $p<0.001$ by ***, and $p<0.0001$ by ***. ("No Rx": No drug). 
(A)

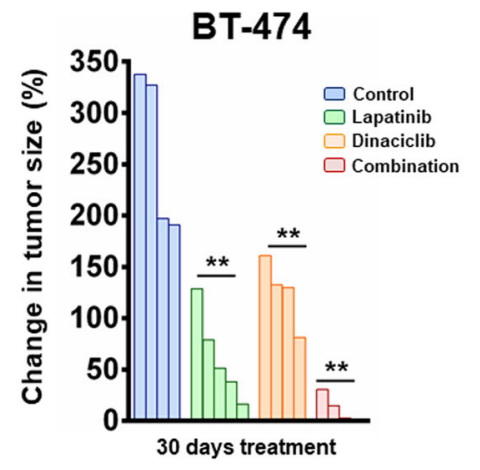

(B)

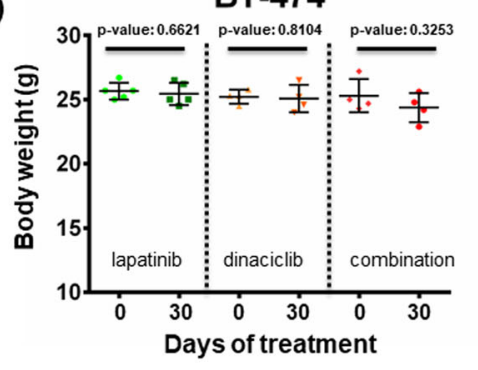

(C)

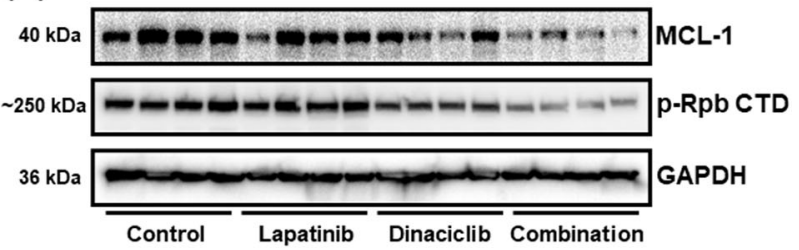

Fig. 4 Combination treatment with lapatinib and dinaciclib leads to anti-tumor activity in vivo. A Approximately, $15 \times 10^{6}$ (15 million) BT-474 cells were injected orthotopically into each NSG mouse (both sides) and monitored for subsequent growth. When tumors were $\sim 200 \mathrm{~mm}^{3}$, mice were randomized into treatment cohorts: control (no drug), $100 \mathrm{mg} / \mathrm{kg}$ lapatinib, $40 \mathrm{mg} / \mathrm{kg}$ dinaciclib, and their combination for 30 days. Dinaciclib was administered twice a week via IP injection. Lapatinib was given orally once a day for 5 consecutive days. Tumor measurements were performed daily, and the percentage (\%) of changes in volume for each tumor is shown by a waterfall plot (control $=4$ tumors, lapatinib $=5$ tumors, dinaciclib $=4$ tumors, combination $=$ 4 tumors). For statistical analysis one-way Anova test was performed for comparisons between lapatinib, dinaciclib, and combination cohorts. Dunnett's test was used as post hoc. Differences were considered statistically different if $p<0.05$. A $p$ value $<0.05$ is indicated by *,$p<0.01$ by **, $p<0.001$ by ***, and $p<0.0001$ by ${ }^{* * *}$. B Weights of the single agents and the combination cohorts of the human xenograft-bearing mice. The number of mice was: control $=5$ mice, lapatinib $=5$ mice, dinaciclib $=4$ mice, and combination $=4$ mice. $p$ values were calculated using the two-tailed Student's $t$ test. C Tumors were harvested from BT474 tumor-bearing mice approximately $2 \mathrm{~h}$ after the last drug administration and tumor lysates were subjected to western blot analyses and probed for the indicated proteins.

dinaciclib exhibited modest efficacy when administered alone but was sufficient to significantly sensitize BT-474 xenografts to lapatinib when dosed twice a week based on the clinical schedule (Fig. 4A and Suplementary Fig. 5A).
Mice remained healthy, based on their weight profiles, treated with the single agents or the combination (Fig. 4B). CDK9 phosphorylates the carboxy-terminal domain (CTD) of the RNA Polymerase II regulating elongation during transcription ${ }^{33}$. Thus, CDK9 inhibitors regulate the expression of proteins with a short half-life, like MCL-1, and the reduction of the phosphorylation of the RNA polymerase II CTD at Ser2 may be used as a biomarker of the activity of CDK9 inhibitors ${ }^{34}$. On-target inhibition of CDK9 was demonstrated by the suppression phosphorylation sites on the CTD of RNA polymerase II as well as MCL-1 following therapy with dinaciclib alone or in combination with lapatinib (Fig. 4C).

\section{Dinaciclib sensitizes neratinib in HER2-amplified patient- derived xenograft (PDX) models}

Neratinib is a potent irreversible pan-HER inhibitor, recently FDA-approved for HER2-amplified breast can$\mathrm{cer}^{2}$. We tested neratinib in combination with dinaciclib in two HER2-amplified PDX models (WHIM 8 and WHIM 22) ${ }^{35}$. While neratinib was effective at blocking the growth of the HER2-amplified tumors, the combination of dinaciclib and neratinib was superior to singleagent therapy in the WHIM 22 model (Fig. 5A and Suplementary Fig, 5B). In addition, there was no weight loss of the mice treated with the single agents or the combination, again suggesting tolerability (Fig. 5B). In the WHIM 8 model, we observed high activity of neratinib monotherapy; however, the combination of neratinib and dinaciclib resulted in uniformly robust tumor shrinkage $(>50 \%)$ (Fig. 5C and Suplementary Fig. 5C), with mice again not showing any significant weight loss (Fig. 5D). Cleaved PARP was elevated when the two drugs were administered together, indicating induction of apoptosis, while reduction of p-HER2 and MCL-1 advocates for the on-target effect of neratinib and dinaciclib, respectively (Fig. 5E). These data demonstrate potent combination efficacy of neratinib and dinaciclib in HER2-positive breast cancer PDX models.

\section{Discussion}

HER2 inhibitors administered in the neo-adjuvant setting increase progression-free survival (the time from treatment initiation until disease progression or worsening) and overall survival (the duration of patient survival from the time of treatment initiation) in HER2amplified breast cancers ${ }^{36,37}$. However, unlike similar RTK inhibitors in other solid tumor paradigms ${ }^{38-40}$ which have now replaced chemotherapy as standard of care, HER2 inhibitors are ineffective as monotherapy. Finding rational targeted therapy combinations with HER2 inhibitors therefore is likely the next step in order to find a therapeutic regimen that does not include chemotherapy. 
(A)

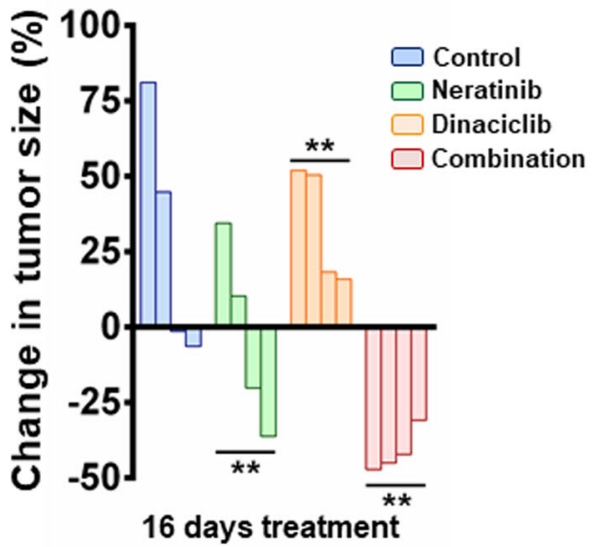

(C)

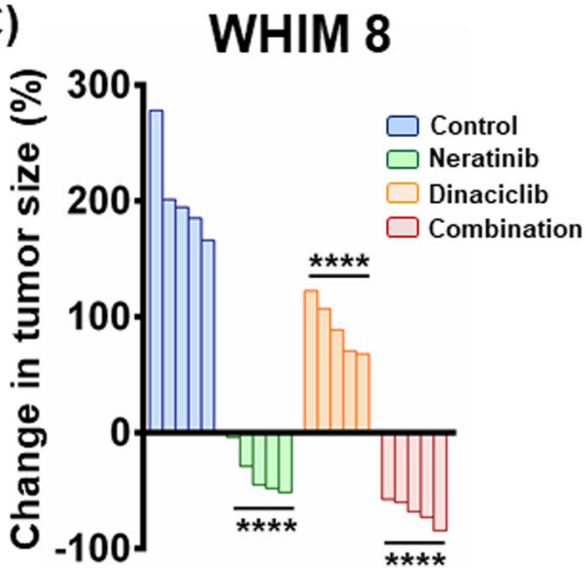

(B)

WHIM 22

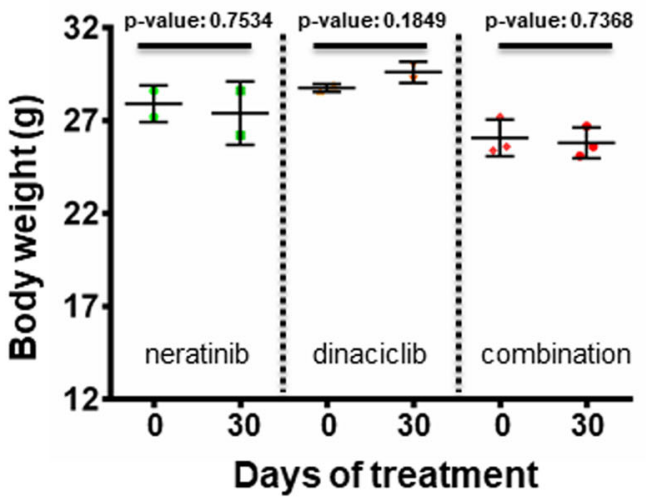

(D)

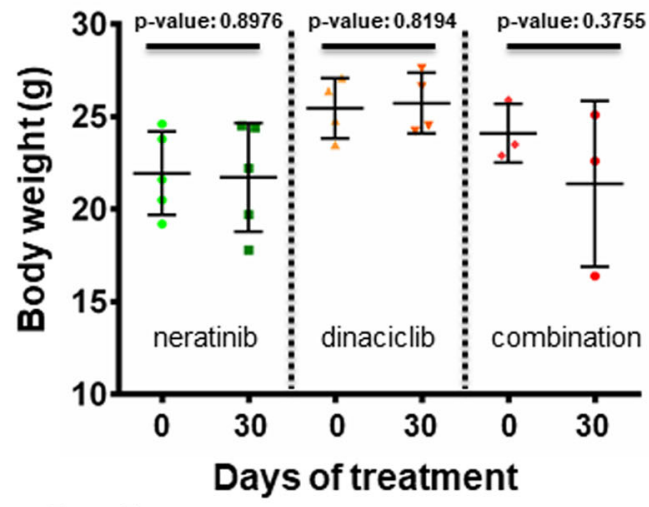

18 days treatment

(E)

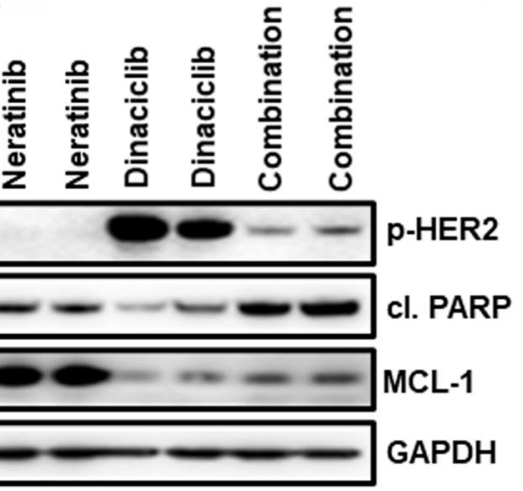

Fig. 5 Combination treatment with neratinib and dinaciclib leads to anti-tumor activity in vivo. A Approximately, $1.5 \times 10^{6}(1.5$ million) cells derived from a HER2-positive breast cancer PDX model (WHIM 22) were injected orthotopically into each NSG mouse (both sides) and monitored for subsequent growth. After tumors reached a size of $\sim 150 \mathrm{~mm}^{3}$, mice were treated with $40 \mathrm{mg} / \mathrm{kg}$ neratinib 5 days a week (Monday-Friday), $40 \mathrm{mg} / \mathrm{kg}$ dinaciclib twice a week, or their combination for 16 days. Tumor measurements were performed every day by calipers, and the percentage (\%) of changes in volume for each tumor is shown by a waterfall plot (control $=4$ tumors, neratinib $=4$ tumors, dinaciclib $=4$ tumors, combination $=4$ tumors). For statistical analysis one-way Anova test was performed for comparisons between neratinib, dinaciclib, and combination cohorts.

Dunnett's test was used as post hoc. Differences were considered statistically different if $p<0.05$. A $p$ value $<0.05$ is indicated by ${ }^{*}, p<0.01$ by ${ }^{*}, p<$ 0.001 by ${ }^{* * *}$, and $p<0.0001$ by ${ }^{* * *}$. B Weights of the WHIM 22 PDX model-bearing mice of the single agents and the combination cohorts. The number of mice was: control $=2$ mice, neratinib $=2$ mice, dinaciclib $=2$ mice, and combination $=3$ mice. $p$ Values were calculated using the twotailed Student's $t$ test. C Same as $\mathbf{A}$ using the WHIM 8, HER2-positive breast cancer PDX model (18 days of treatment, control $=5$ tumors, neratinib $=$ 5 tumors, dinaciclib $=4$ tumors, combination $=3$ tumors). D Same as $\mathbf{B}$ using the WHIM 8 PDX model. The number of mice was: control $=5$ mice, neratinib $=5$ mice, dinaciclib $=2$ mice, and combination $=3$ mice. $\mathbf{E}$ Tumors were harvested from WHIM 8 PDX tumor-bearing mice approximately $2 \mathrm{~h}$ after the last drug administration and tumor lysates were subjected to western blot analyses and probed for the indicated proteins. 
Indeed, chemotherapy has already begun to be deemphasized in breast cancer, in particular hormone positive breast cancer ${ }^{41}$. The reason for de-escalation is the broad and lasting effects of chemotherapy-induced toxicity, which has been well described in breast cancer. Toxicities are numerous and cover a wide range of tissues. Cardiac toxicity, including congestive heart failure, is contributed by anthracyclines like doxorubicin ${ }^{42}$. Reproductive toxicity is very common for breast cancer undergoing adjuvant chemotherapy: for instance, in 280 young (aged 24-45) breast cancer patients, over 90\% suffered from chemotherapy-related amenorrhea ${ }^{43}$. While there remains controversy, a large Swedish study demonstrated women treated with chemotherapy for their breast cancer had higher risk pregnancies ${ }^{44}$. Chemotherapy-induced bone loss is also a significant toxicity with considerable morbidity ${ }^{45,46}$. In addition to overt tissue toxicity, chemotherapy delivered during breast cancer treatment increases the risk of secondary cancers, in particular acute myeloid leukemia ${ }^{47,48}$.

Recently, we reported that levels of the endogenous MCL-1 inhibitor, NOXA, are uniformly depressed in HER2-amplified breast cancers, as a result of a coamplified intronic microRNA that targets the estrogen receptor (ER), which in turn leads to loss of ER-driven NOXA transcription ${ }^{4}$. This can be overcome by the addition of MCL-1 BH3 mimetics, which Merino et al. ${ }^{5}$ also demonstrated. However, the toxicity of these drugs in clinical trials remains to be defined. Interestingly, we also found co-targeting BCL-xL with HER2 is effective (Suplementary Fig. 4A, C), verifying results that have previously been reported ${ }^{25}$. In Fig. 2 and Suplementary Fig. 3B, D, we provide evidence that dinaciclib and consequently its combination with lapatinib target mainly MCL-1. However, in SKBR3 cells overexpression of BCL$\mathrm{xL}$ partially rescues sensitivity to dinaciclib and its combination with lapatinib (Suplementary Fig. 3B), albeit to a smaller extent than overexpression of MCL-1 does (Fig. 2D). This could be explained by the subsequent binding of the freed BAK to BCL-xL that is supplied exogenously, for which BAK has also affinity ${ }^{23}$. While small molecule BCL-xL inhibitors have so far proven too toxic $^{49,50}$, other strategies to target BCL-xL, for instance, PROTACS, are being developed ${ }^{51}$. Indeed, Brugge and colleagues demonstrated potent preclinical in vivo activity of the dual BCL-xL/BCL-2 inhibitor navitoclax with the HER2-targeting antibody-drug conjugate trastuzumab emtansine ${ }^{52}$.

In contrast to the fairly unknown toxicity of MCL-1 inhibitors, dinaciclib is a CDK1, 2, 5, and 9 inhibitor that has demonstrated limited toxicities as a monotherapy, many of which were transient ${ }^{6,53}$. CDK9 is part of the CAK complex, which is responsible for phosphorylating the C-terminus of RNA polymerase II, regulating elongation during transcription ${ }^{33}$. Although there are other cyclin-dependent kinases that are capable of phosphorylating the CTD of the RNA Polymerase II, like CDK7 and CDK8, the only one that activates gene expression in a catalytic manner is CDK9 ${ }^{54}$. CDK9 inhibitors regulate the expression of proteins with a short half-life. In this context dinaciclib has been reported to suppress the expression levels of the homologous recombination (HR) repair factors Rad51 and BRCA1 as well as $\mathrm{c}-\mathrm{Myc}^{55,56}$. Notwithstanding the fact that MCL-1 is not the only protein that is downregulated after treatment with dinaciclib, the lack of its pro-apoptotic partner, NOXA, in HER2-amplified breast cancers ${ }^{4}$ makes it likely the most important dinaciclib target in HER2-amplified breast cancers. Of note, there are other CDK inhibitors that have been explored for the treatment of HER2amplified breast cancers, but no correlation with the expression of MCL-1 has been established ${ }^{57}$.

Combining HER2 inhibitors with a targeted therapy that can sensitize to apoptosis is an important therapeutic strategy since a robust apoptosis response is essential for mono-therapeutic targeted therapy in other RTK-driven cancers $^{58-60}$. In fact, in paradigms such as EGFR-mutant NSCLC, EGFR inhibition has limited success in patients whose cancers cannot undergo robust apoptosis ${ }^{58,61-65}$. We believe the ability of dinaciclib to rationally combine with HER2 inhibitors to induce apoptosis could therefore overcome the lack of efficacy HER2 inhibitors in HER2amplified breast cancers display, providing a targeted therapy combination strategy that could potentially eliminate the need for chemotherapy.

Since in addition to forming complexes with proapoptotic BCL-2 family members, MCL-1 also exerts oncogenic activity through other means ${ }^{66,67}$, pharmaceutical reduction of MCL-1 expression may be more broadly effective than exposure to MCL-1 BH3 mimetics. Indeed, we noted increased sensitivity of dinaciclib and lapatinib compared to A-1210477 and lapatinib (Fig. 1). In addition, it should be noted that both lapatinib and neratinib are considered dual inhibitors of HER2 and EGFR ${ }^{68,69}$, which contributes to dermatologic and gastrointestinal adverse events $^{70,71}$. We also investigated the efficacy of the highly selective HER2 inhibitor tucatinib combined with CDK9 inhibition. Consistently, our data support the notion that combination treatment of dinaciclib with selective HER2 inhibition can be an effective therapy against HER2amplified breast cancer.

In all, we propose that treating $H E R 2$-positive breast cancers by co-targeting HER2 and MCL- 1 can be achieved with the CDK inhibitor dinaciclib, which is clinically advanced. This combination may have advantages over MCL-1 BH3 mimetics, therefore maximizing the potential of HER2 inhibitors to treat HER2-amplified breast cancers. Importantly, this offers a strategy that is 
independent of chemotherapy, with the aim of improving responses and decreasing toxicity.

\section{Materials and methods Cell lines}

The HER2-positive breast cancer cell lines used in this study were kindly provided by the Massachusetts General Hospital. SKBR3 cells were grown in DMEM/F12 medium with $10 \%$ fetal bovine serum (FBS) in the presence of $1 \mu \mathrm{g} /$ $\mathrm{mL}$ penicillin and streptomycin. BT-474 cells were cultured in DMEM medium containing 10\% FBS, $1 \mu \mathrm{g} / \mathrm{ml}$ penicillin, streptomycin, and $5 \mu \mathrm{g} / \mathrm{ml}$ of insulin. MDAMB-453, HCC-1419 were cultured in RPMI with 10\% FBS in the presence of $1 \mu \mathrm{g} / \mathrm{mL}$ penicillin and streptomycin. Cells were regularly screened for mycoplasma using a MycoAlert Mycoplasma Detection Kit (Lonza).

\section{Reagents}

The following drugs were purchased: Dinaciclib (SCH727965) for in vitro and in vivo studies (S2768; Selleckchem), lapatinib ditosylate (Tykerb) for in vitro and in vivo studies (M1802; Abmole), neratinib for in vivo studies (M1913; Abmole), A-1210477 (CT-A121; Chemietek), A-1331852 (22963; Cayman Chemicals), tucatinib (HY-16069; Medchem), and ABT-199 (venetoclax) (CT-A199; Chemietek). The antibodies used in this study were as follows: Anti-Bak (AB-1 clone for IP) (AM03; EMD Millipore), anti-Bak (3814S; Cell Signaling), antiBim (C34C5) (2933S; Cell Signaling), anti-BCL-xL (54H6) (2764S; Cell Signaling), anti-Bcl-2 (D55G8) (Human Specific) (4223S; Cell Signaling), anti-cleaved PARP (Asp214) (D64E10) (5625S; Cell Signaling), antiGAPDH (6C5) (sc-32233; Santa Cruz), anti-MCL-1 (S19) (sc-819; Santa Cruz), anti-phospho-p44/42 MAPK (Erk1/2) (Thr202/Tyr204) (D13.14.4E) (4370S; Cell Signaling), anti-phospho-S6 Ribosomal Protein (Ser240/244) (D68F8) (5364S; Cell Signaling), anti-phospho-Akt (Thr308) (244F9) (4056S; Cell Signaling), anti-phosphoAkt (Ser473) (D9E) (4060S; Cell Signaling), anti-HER2/ ErbB2 (29D8) (2165S; Cell Signaling), anti-phosphoHER2/ErbB2 (Tyr1248) (2247S; Cell Signaling), antiphospho-Rpb1 CTD (Ser 2/5) (4375S; Cell Signaling), Normal Rabbit IgG for IP (sc-2027; Santa Cruz), and Normal Mouse IgG for IP (sc-2025; Santa Cruz).

\section{Vector construction and establishing stable cell lines}

For the short-hairpin RNA (shRNA) experiments, the lentiviral shRNA (shBAK) was purchased from Open Biosystems. shRNA designed against a scramble sequence (MISSION pLKO.1-shRNA control plasmid DNA) served as the control. The pLKO.1 puromycin-resistant vector backbone served as the basis for cell selection in puromycin following infection. Cells were transduced with plasmid containing viral particles that were generated in
293T cells and collected over $48 \mathrm{~h}$. The human MCL1 expression vector was generated as previously described (2). The construct was transfected into $293 \mathrm{~T}$ packaging cells along with the packaging plasmids and the lentiviruscontaining supernatants were collected to transduce the cells.

\section{Western blotting}

Cell lines and tumors from BT-474 xenografts as well as PDXs were prepared and lysed in lysis buffer $(20 \mathrm{mM}$ Tris, $150 \mathrm{mM} \mathrm{NaCl}, 1 \%$ Nonidet P-40, $1 \mathrm{mM}$ EDTA, $1 \mathrm{mM}$ EGTA, 10\% glycerol, and protease, and phosphatase inhibitors), incubated on ice for $15 \mathrm{~min}$, and centrifuged at max speed for $10 \mathrm{~min}$ at $4{ }^{\circ} \mathrm{C}$. Tumor lysates were homogenized with Tissuemiser (Fisher Scientific) in the lysis buffer described previously, incubated for $20 \mathrm{~min}$ on ice, and centrifuged at max speed for $10 \mathrm{~min}$ at $4{ }^{\circ} \mathrm{C}$. Equal amounts of the detergent-soluble lysates were resolved using the NuPAGE Novex Midi Gel system on 4-12\% Bis-Tris gels (Invitrogen), transferred to polyvinylidene fluoride membranes (PerkinElmer) in between six pieces of Whatman paper (Fisher Scientific) set in transfer buffer from Biorad with $20 \%$ methanol, and following transfer and blocking in 5\% nonfat milk in PBS, probed overnight with the antibodies listed above. Representative blots from at least three independent experiments are shown in the figures. Chemiluminescence was detected with the Syngene G: Box camera (Synoptics).

\section{Cell viability assay}

For the Cell Titer-Glo experiments, 1000-3000 seeded cells per well in 96-well flat-bottom black plates were treated with $25 \mu \mathrm{L}$ of CellTiter-Glo (Promega), following continuous drug treatment (each time with the indicated drugs at the indicated concentrations), at $37^{\circ}$ and $5 \%$ atmospheric $\mathrm{CO}_{2}$ and immediately read on a Centro LB 960 microplate luminometer (Berthold Technologies) according to the Promega protocol. Quantification of notreatment seeded cells was used to determine the total cell growth number over the experiment. All data are means $\pm \mathrm{SD}$ of three independent experiments $(n=3)$.

\section{FACS apoptosis assay}

Totally, $3 \times 10^{5}$ cells were seeded per well in six-well plates and drugged with $100 \mathrm{nM}$ dinaciclib combined with $1 \mu \mathrm{M}$ lapatinib for 24 (BT-474) and $72 \mathrm{~h}$ (MDA-MB-453), or left untreated. Cells were incubated with propidium iodide and annexin V-Cy5 (BD Biosciences) together for $15 \mathrm{~min}$ and assayed on a Guava easyCyte 5 flow cytometer (Millipore Sigma). Analysis was performed using guavaSoft 3.1.1 software. Cells stained positive for annexin V and annexin $\mathrm{V}+$ propidium iodide were counted as apoptotic. All data are means \pm SD of three independent experiments $(n=3)$. 


\section{RNA extraction and qRT-PCR}

RNA was isolated from cultured cells grown at subconfluency using the Zymo Quick-RNA MiniPrep kit (Zymo Research), and RNA was reverse-transcribed to form cDNA molecules using cDNA synthesis kit superscript III (Invitrogen) on a 7500 Fast Real-Time PCR System (Life Technologies). The expression of $M C L-1$, and $\beta$-ACTIN $(A C T B)$ was measured using a GENEAMP PCR System 9700 (Life Technologies) by measuring the fluorescence increases of SYBR Green (Roche). The primers for $M C L-1$ forward $5^{\prime}$-GGGCAGGATTGTGACTC TCATT-3' and $M C L-1$ reverse 5'-GATGCAGCTTTC TTGGTTTATGG- $3^{\prime}$ and for $A C T B$ forward $5^{\prime}$-GGCAT GGGTCAGAAGGATT- $3^{\prime}$, and $A C T B$ reverse $5^{\prime}$-AGGAT GCCTCTCTTGCTCTG-3'. To determine relative abundance of $M C L-1$ in relation to $A C T B$, the Delta-Delta CT (cycle threshold) method was utilized. All data are means + SEM of three independent experiments $(n=3)$.

\section{Immunoprecipitation}

Cells were lysed in the same buffer above; $500 \mu \mathrm{g}$ of lysates were incubated each time with MCL-1 antibody (2000 ng), or rabbit IgG (2000 ng). Following the addition of $25 \mu \mathrm{L}$ of 1:1 PBS: prewashed Protein A Sepharose CL-4B beads (cat. no. 17-096303; GE Healthcare Life Sciences) to the antibody/lysate mix, samples were incubated with rotating motion overnight. Equal amounts of extracts (5\% of immunoprecipitated protein) were also prepared. Representative blots from at least three independent experiments are shown in the figures. Chemiluminescence was detected with the Syngene G: Box camera (Synoptics).

\section{BAK activation assay}

Cells were treated as indicated and lysed in AB- 1 amino terminal capture buffer (10 mM Hepes, $135 \mathrm{mM} \mathrm{NaCl}, 5 \mathrm{mM}$ $\mathrm{MgCl}_{2}, 0.2 \mathrm{mM}$ EDTA, $1 \%$ glycerol + 1\% CHAPS, added fresh; $\mathrm{pH} 7.4) ; 1500 \mu \mathrm{g}$ of lysates for the assay were incubated each time with $\mathrm{AB}-1 / \mathrm{BAK}$ antibody (1000 ng). Following the addition of $25 \mu \mathrm{L}$ of 1:1 PBS: prewashed Protein A Sepharose CL-4B beads (cat. no. 17-0963-03; GE Healthcare Life Sciences) to the antibody/lysate mix, samples were incubated with rotating motion overnight. Equal amounts of extracts (2.5\% of immunoprecipitated protein) were also prepared. Representative blots from at least three independent experiments are shown in the figures. Chemiluminescence was detected with the Syngene G: Box camera (Synoptics).

\section{Xenograft studies}

NSG female mice were injected with $\sim 15 \times 10^{6}$ BT- 474 cells per $200 \mu \mathrm{L}$ of 1:1 (cells: Matrigel). Mice were injected intraductally both sides and monitored for tumor growth. When tumors reached $\sim 200 \mathrm{~mm}^{3}$, the tumor-bearing mice were randomized to a no-treatment control group, a lapatinib group $(100 \mathrm{mg} / \mathrm{kg})$, a dinaciclib group $(40 \mathrm{mg} /$ $\mathrm{kg}$ ), or a combination group (same doses). Mice in the cohorts (control $=4$ tumors, lapatinib $=5$ tumors, dinaciclib $=4$ tumors, combination $=4$ tumors) were treated with dinaciclib via IP injection and $2 \mathrm{~h}$ later with lapatinib by oral gavage. The solvent for lapatinib was $1 \%$ Tween 80. Dinaciclib was formulated in 20\% 2-hydroxy propyl$\beta$-cyclo dextrin (Sigma-Aldrich). The tumors were measured daily by electronic caliper, in two dimensions (length and width), and with the formula $v=1 \times(w) 2(\pi / 6)$, where $v$ is the tumor volume, $l$ is the length, and $w$ is the width (the smaller of the two measurements). The drug schedule was 5 days a week (Monday-Friday) for lapatinib and twice a week for dinaciclib for 30 days. For pharmacodynamic studies, tumors were harvested $2 \mathrm{~h}$ following the last lapatinib treatment, and tumors were snap frozen in liquid nitrogen. All mouse experiments were approved and performed in accordance with the Institutional Animal Care and Use Committee at VCU.

\section{Patient-derived xenografts}

Female NSG mice were inoculated with tumor pieces derived from two HER2 + breast cancer PDX models called WHIM 8 and WHIM 22 (Horizon Discovery Group $^{35}$, expanded as single cell suspensions and injected into experimental mice orthotopically at the amount indicated in the legend of Fig. 4. Tumor growth was monitored until tumors grew to treatable levels $\left(\sim 150 \mathrm{~mm}^{3}\right)$. These mice were then randomized into four groups: control, neratinib $(40 \mathrm{mg} / \mathrm{kg})$, dinaciclib $(40 \mathrm{mg} / \mathrm{kg})$, and dinaciclib/neratinib combination treatment. The number of tumors per cohort was: $\operatorname{control}=4$ tumors, neratinib $=4$ tumors, dinaciclib $=4$ tumors, combination $=4$ tumors for the WHIM 22 model and control $=5$ tumors, neratinib $=5$ tumors, dinaciclib $=5$ tumors, combination $=5$ tumors for the WHIM 8 model. Dinaciclib was formulated in 20\% 2-hydroxy propyl- $\beta$-cyclo dextrin (Sigma-Aldrich), while the solvent for neratinib was $0.5 \%$ methocellulose $-0.4 \%$ Tween 80. Mice in the cohorts were treated with dinaciclib via IP injection and $2 \mathrm{~h}$ later with neratinib by oral gavage. The drug schedule was 5 days a week (Monday-Friday) for neratinib and twice a week for dinaciclib for 16 days (WHIM 22) or 18 days (WHIM 8). For pharmacodynamic studies, tumors were harvested $2 \mathrm{~h}$ following the last neratinib treatment, and tumors were snap frozen in liquid nitrogen. Tumors were measured as per the BT474 xenograft.

\section{Statistical considerations}

Two-tailed Student's $t$ test was performed for Figs. 1B-D, 2D, F, 3B, D, Suplementary Fig. 1, Suplementary Fig. 3B, D, Suplementary Fig. 4 and Suplementary Fig. 5A-C using GraphPad Prism. $p$ values were corrected for multiple testing using Bonferroni method. 
For Figs. 4A, 5A and 5C one-way Anova test was performed for comparisons between lapatinib/neratinib, dinaciclib and combination cohorts. Dunnett's test was used as post hoc. Differences were considered statistically different if $p<0.05$. A $p$ value $<0.05$ is indicated by $*, p<$ 0.01 by ${ }^{* *}, p<0.001$ by ${ }^{* * * *}$, and $p<0.0001$ by ${ }^{* * * * * *}$.

\section{Acknowledgements}

Services and products in support of the research project were generated by the Virginia Commonwealth University Cancer Mouse Models Core Laboratory, supported, in part, with funding to the Massey Cancer Center from $\mathrm{NIH}-\mathrm{NCl}$ Cancer Center Support Grant P30 CA016059.

\section{Funding}

This study was supported by the Department of Defense Breast Cancer Research Program, W81XWH-18-1-0561 (M.S. and A.C.F.) the Breast Cancer Research Foundation and the Mary Kay Foundation.

\section{Author details \\ 'Department of Oral and Craniofacial Molecular Biology, Philips Institute for Oral Health Research, VCU School of Dentistry and Massey Cancer Center, Virginia Commonwealth University, Richmond, VA 23298, USA. ${ }^{2}$ Department of Pathology, Virginia Commonwealth University School of Medicine and Massey Cancer Center, Richmond, VA 23220, USA. ${ }^{3}$ Department of Biostatistics, Virginia Commonwealth University, Richmond, VA 23298, USA. ${ }^{4}$ Division of Hematology, Oncology and Palliative Care, Virginia Commonwealth University and Massey Cancer Center, Richmond, VA 23298, USA. ${ }^{5}$ AstraZeneca Pharmaceuticals, 35 Gatehouse Dr., Waltham, MA 02451, USA. ${ }^{6}$ Human Oncology \& Pathogenesis Program (HOPP), Memorial Sloan Kettering Cancer Center, New York, NY 10065, USA. ${ }^{7}$ Department of Pathology, Memorial Sloan Kettering Cancer Center, New York, NY 10065, USA}

\section{Author contributions}

K.V.F., M.S., and A.C.F. performed the study concept and design; K.V.F. and A.C.F. performed the development of methodology and writing, review, and revision of the paper; K.V.F., S.J., R.K., C.K.F., B.H., M.P., J.K., M.G.D., S.A.B., and A.C.F. provided the acquisition, analysis and interpretation of data, and statistical analysis; all authors read and approved the final paper.

\section{Conflict of interest}

In the past 2 years M.S. has received funds from Puma Biotechnology, AstraZeneca, Daiichi-Sankio, Immunomedics, Targimmune and Menarin Ricerche, and is a cofounder of Medendi.org and a full employee of AstraZeneca. A.F. has served as a scientific advisor for AbbVie, Inc.

\section{Ethics statement}

The current study did not require ethical approval.

\section{Publisher's note}

Springer Nature remains neutral with regard to jurisdictional claims in published maps and institutional affiliations.

Supplementary information The online version contains supplementary material available at https://doi.org/10.1038/s41419-021-03457-6.

Received: 11 April 2020 Revised: 23 December 2020 Accepted: 3 January 2021

Published online: 15 February 2021

\section{References}

1. Gianni, L. et al. Neoadjuvant chemotherapy with trastuzumab followed by adjuvant trastuzumab versus neoadjuvant chemotherapy alone, in patients with HER2-positive locally advanced breast cancer (the NOAH trial): a randomised controlled superiority trial with a parallel HER2-negative cohort. Lancet 375, 377-384 (2010).
2. Chan, A. et al. Neratinib after trastuzumab-based adjuvant therapy in patients with HER2-positive breast cancer (ExteNET): a multicentre, randomised, double-blind, placebo-controlled, phase 3 trial. Lancet Oncol. 17, 367-377 (2016)

3. Lynch, T. J. et al. Novel agents in the treatment of lung cancer: Fourth Cambridge Conference. Clin. Cancer Res. 13, s4583-s4588 (2007).

4. Floros, K. V. et al. Coamplification of miR-4728 protects HER2-amplified breast cancers from targeted therapy. Proc. Natl. Acad. Sci. USA 115, E2594-e2603 (2018).

5. Merino, D. et al. Synergistic action of the MCL-1 inhibitor $\$ 63845$ with current therapies in preclinical models of triple-negative and HER2-amplified breast cancer. Sci. Transl. Med. 9, eaam7049 (2017).

6. Thomas, D. et al. Targeting acute myeloid leukemia by dual inhibition of PI3K signaling and Cdk9-mediated Mcl-1 transcription. Blood 122, 738-748 (2013).

7. Hsieh, A. C. et al. Genetic dissection of the oncogenic mTOR pathway reveals druggable addiction to translational control via 4EBP-elF4E. Cancer Cell 17, 249-261 (2010).

8. She, Q. B. et al. 4E-BP1 is a key effector of the oncogenic activation of the AKT and ERK signaling pathways that integrates their function in tumors. Cancer Cell 18, 39-51 (2010).

9. Fu, W. et al. The cyclin-dependent kinase inhibitor SCH 727965 (dinacliclib) induces the apoptosis of osteosarcoma cells. Mol. Cancer Therap. 10, 1018-1027 (2011).

10. $\mathrm{Xu}$, J. et al. Inhibition of cyclin E1 sensitizes hepatocellular carcinoma cells to regorafenib by mcl-1 suppression. Cell Commun. Signal. 17, 85 (2019).

11. Gregory, G. P. et al. CDK9 inhibition by dinaciclib potently suppresses Mcl-1 to induce durable apoptotic responses in aggressive MYC-driven B-cell lymphoma in vivo. Leukemia 29, 1437-1441 (2015).

12. Jane, E. P. et al. Dinaciclib, a cyclin-dependent kinase inhibitor promotes proteasomal degradation of Mcl-1 and enhances ABT-737-mediated cell death in malignant human glioma cell lines. J. Pharmacol. Exp. Therap. 356, 354-365 (2016).

13. $L i$, L. et al. Synergistic induction of apoptosis in high-risk DLBCL by $B C L 2$ inhibition with ABT-199 combined with pharmacologic loss of MCL1. Leukemia 29, 1702-1712 (2015).

14. Song, K. A. et al. Increased synthesis of MCL-1 protein underlies initial survival of EGFR-mutant lung cancer to EGFR inhibitors and provides a novel drug target. Clin. Cancer Res. 24, 5658-5672 (2018).

15. Gojo, I. et al. Clinical and laboratory studies of the novel cyclin-dependent kinase inhibitor dinaciclib (SCH 727965) in acute leukemias. Cancer Chemother. Pharmacol. 72, 897-908 (2013).

16. Mita, M. M. et al. Randomized phase II trial of the cyclin-dependent kinase inhibitor dinaciclib (MK-7965) versus capecitabine in patients with advanced breast cancer. Clin. Breast Cancer 14, 169-176 (2014).

17. Faber, A. C. et al. Differential induction of apoptosis in HER2 and EGFR addicted cancers following PI3K inhibition. Proc. Natl. Acad. Sci. USA 106, 19503-19508 (2009).

18. Zhou, J. et al. AMPK mediates a pro-survival autophagy downstream of PARP1 activation in response to DNA alkylating agents. FEBS Lett. 587, 170-177 (2013).

19. Yao, M. et al. The research on lapatinib in autophagy, cell cycle arrest and epithelial to mesenchymal transition via Wnt/ErK/PI3K-AKT signaling pathway in human cutaneous squamous cell carcinoma. J. Cancer 8, 220-226 (2017).

20. Erlich, S. et al. Differential interactions between Beclin 1 and Bcl-2 family members. Autophagy 3, 561-568 (2007).

21. Huang, H., Shah, K, Bradbury, N. A., Li, C. \& White, C. Mcl-1 promotes lung cancer cell migration by directly interacting with VDAC to increase mitochondrial $\mathrm{Ca} 2+$ uptake and reactive oxygen species generation. Cell Death Dis. 5, e1482 (2014)

22. Ham, J. et al. Exploitation of the apoptosis-primed state of MYCN-amplified neuroblastoma to develop a potent and specific targeted therapy combination. Cancer cell 29, 159-172 (2016).

23. Willis, S. N. et al. Proapoptotic Bak is sequestered by Mcl-1 and BCl-xL, but not BCl-2, until displaced by BH3-only proteins. Genes Dev. 19, 1294-1305 (2005).

24. Griffiths, G. J. et al. Cell damage-induced conformational changes of the proapoptotic protein Bak in vivo precede the onset of apoptosis. J. Cell Biol. 144, 903-914 (1999).

25. Xiao, Y. et al. MCL-1 is a key determinant of breast cancer cell survival: validation of MCL-1 dependency utilizing a highly selective small molecule inhibitor. Mol. Cancer Therap. 14, 1837-1847 (2015). 
26. Choong, G. M., Cullen, G. D. \& O'Sullivan, C. C. Evolving standards of care and new challenges in the management of HER2-positive breast cancer. CA Cancer J. Clin. 70, 355-374 (2020).

27. Kulukian, A. et al. Preclinical activity of HER2-selective tyrosine kinase inhibitor tucatinib as a single agent or in combination with trastuzumab or docetaxel in solid tumor models. Mol. Cancer Therap. 19, 976-987 (2020).

28. Shah, M. et al. FDA approval summary: tucatinib for the treatment of patients with advanced or metastatic HER2-positive breast cancer. Clin. Cancer Res. (2020).

29. Tucatinib Is Active Against Brain Metastases in HER2(+) Breast Cancer. Cancer Discov. 10, 1090 (2020).

30. Lin, N. U. et al. Intracranial efficacy and survival with tucatinib plus trastuzumab and capecitabine for previously treated HER2-positive breast cancer with brain metastases in the HER2CLIMB trial. J. Clin. Oncol. 38, 2610-2619 (2020).

31. Murthy, R. K. et al. Tucatinib, Trastuzumab, and Capecitabine for HER2-Positive Metastatic Breast Cancer. N. Engl. J. Med. 382, 597-609 (2020).

32. Tucatinib Impresses in Breast Cancer. Cancer Discov. 10, 7 (2020).

33. Bieniasz, P. D., Grdina, T. A., Bogerd, H. P. \& Cullen, B. R. Recruitment of cyclin T1/P-TEFb to an HIV type 1 long terminal repeat promoter proximal RNA target is both necessary and sufficient for full activation of transcription. Proc. Natl. Acad. Sci. USA 96, 7791-7796 (1999).

34. Natoni, A. et al. Mechanisms of action of a dual Cdc7/Cdk9 kinase inhibitor against quiescent and proliferating CLL cells. Mol. Cancer Therap. 10 1624-1634 (2011).

35. Li, S. et al. Endocrine-therapy-resistant ESR1 variants revealed by genomic characterization of breast-cancer-derived xenografts. Cell Rep. 4, 1116-1130 (2013).

36. Slamon, D. J. et al. Use of chemotherapy plus a monoclonal antibody against HER2 for metastatic breast cancer that overexpresses HER2. N. Engl. J. Med. 344, 783-792 (2001)

37. Piccart-Gebhart, M. J. et al. Trastuzumab after adjuvant chemotherapy in HER2positive breast cancer. N. Engl. J. Med. 353, 1659-1672 (2005).

38. Kwak, E. L. et al. Anaplastic lymphoma kinase inhibition in non-small-cell lung cancer. N. Engl. J. Med. 363, 1693-1703 (2010).

39. Sequist, L. V. et al. First-line gefitinib in patients with advanced non-small-cell lung cancer harboring somatic EGFR mutations. J. Clin. Oncol. 26, 2442-2449 (2008).

40. Chapman, P. B. et al. Improved survival with vemurafenib in melanoma with BRAF V600E mutation. N. Engl. J. Med. 364, 2507-2516 (2011).

41. Sparano, J. A. et al. Adjuvant chemotherapy guided by a 21-gene expression assay in breast cancer. N. Engl. J. Med. 379, 111-121 (2018).

42. Bird, B. R. \& Swain, S. M. Cardiac toxicity in breast cancer survivors: review of potential cardiac problems. Clin. Cancer Res. 14, 14-24 (2008).

43. Liem, G. S. et al. Chemotherapy-related amenorrhea and menopause in young chinese breast cancer patients: analysis on incidence, risk factors and serum hormone profiles. PLOS ONE 10, e0140842 (2015).

44. Dalberg, K., Eriksson, J. \& Holmberg, L. Birth outcome in women with previously treated breast cancer-a population-based cohort study from Sweden. PLoS Med. 3, e336 (2006)

45. Gnant, M. F. et al. Zoledronic acid prevents cancer treatment-induced bone loss in premenopausal women receiving adjuvant endocrine therapy for hormone-responsive breast cancer: a report from the Austrian Breast and Colorectal Cancer Study Group. J. Clin. Oncol. 25, 820-828 (2007).

46. Brufsky, A. Management of cancer-treatment-induced bone loss in postmenopausal women undergoing adjuvant breast cancer therapy: a Z-FAST update. Semin. Oncol. 33, S13-S17 (2006).

47. Rosenstock, A. S. et al. Acute myeloid leukemia and myelodysplastic syndrome after adjuvant chemotherapy: a population-based study among older breast cancer patients. Cancer 124, 899-906 (2018).

48. Smith, R. E. et al. Acute myeloid leukemia and myelodysplastic syndrome after doxorubicin-cyclophosphamide adjuvant therapy for operable breast cancer: the National Surgical Adjuvant Breast and Bowel Project Experience. J. Clin Oncol. 21, 1195-1204 (2003)

49. Kaefer, A. et al. Mechanism-based pharmacokinetic/pharmacodynamic metaanalysis of navitoclax (ABT-263) induced thrombocytopenia. Cancer Chemother. Pharmacol. 74, 593-602 (2014).

50. Mason, K. D. et al. Programmed anuclear cell death delimits platelet life span Cell 128, 1173-1186 (2007).

51. Khan, $\mathrm{S}$. et al. A selective $B C L-X(L)$ PROTAC degrader achieves safe and potent antitumor activity. Nat. Med. 25, 1938-1947 (2019).

52. Zoeller, J. J. et al. Neutralization of $B C L-2 / X(L)$ enhances the cytotoxicity of $T-$ DM1 in vivo. Mol. Cancer Therap. 18, 1115-1126 (2019).

53. Kumar, S. K. et al. Dinaciclib, a novel CDK inhibitor, demonstrates encouraging single-agent activity in patients with relapsed multiple myeloma. Blood $\mathbf{1 2 5}$, 443-448 (2015).

54. Napolitano, G., Majello, B., Licciardo, P., Giordano, A. \& Lania, L. Transcriptional activity of positive transcription elongation factor $b$ kinase in vivo requires the C-terminal domain of RNA polymerase II. Gene 254, 139-145 (2000).

55. Carey, J. P. W. et al. Synthetic lethality of PARP inhibitors in combination with MYC blockade is independent of BRCA status in triple-negative breast cancer. Cancer Res. 78, 742-757 (2018).

56. Alagpulinsa, D. A., Ayyadevara, S., Yaccoby, S. \& Shmookler Reis, R. J. A cyclindependent kinase inhibitor, dinaciclib, impairs homologous recombination and sensitizes multiple myeloma cells to PARP inhibition. Mol. Cancer Therap. 15, 241-250 (2016).

57. Sun, B. et al. Inhibition of the transcriptional kinase CDK7 overcomes therapeutic resistance in HER2-positive breast cancers. Oncogene 39, 50-63 (2020).

58. Faber, A. C. et al. BIM expression in treatment-naive cancers predicts responsiveness to kinase inhibitors. Cancer Discov. 1, 352-365 (2011).

59. Roulston, A., Muller, W. J. \& Shore, G. C. BIM, PUMA, and the achilles' heel of oncogene addiction. Sci. Signal. 6, pe12 (2013).

60. Bean, G. R. et al. PUMA and BIM are required for oncogene inactivationinduced apoptosis. Sci. Signal. 6, ra20 (2013).

61. Wu, S. G., Liu, Y. N., Yu, C. J., Yang, P. C. \& Shih, J. Y. Association of BIM deletion polymorphism with intrinsic resistance to EGFR tyrosine kinase inhibitors in patients with lung adenocarcinoma. JAMA Oncol. 2, 826-828 (2016).

62. Huang, W. F. et al. BIM gene polymorphism lowers the efficacy of EGFR-TKls in advanced nonsmall cell lung cancer with sensitive EGFR mutations: a systematic review and meta-analysis. Medicines $\mathbf{9 4}$, e1263 (2015).

63. Su, W. et al. BIM deletion polymorphism predicts poor response to EGFR-TKIs in nonsmall cell lung cancer: an updated meta-analysis. Medicines 98, e14568 (2019).

64. Cardona, A. F. et al. BIM deletion polymorphisms in Hispanic patients with non-small cell lung cancer carriers of EGFR mutations. Oncotarget 7, 68933-68942 (2016).

65. Karachaliou, N. et al. BIM and mTOR expression levels predict outcome to erlotinib in EGFR-mutant non-small-cell lung cancer. Sci. Rep. 5, 17499 (2015).

66. Morciano, G. et al. Mcl-1 involvement in mitochondrial dynamics is associated with apoptotic cell death. Mol. Biol. Cell 27, 20-34 (2016).

67. Lee, K. M. et al. MYC and MCL1 cooperatively promote chemotherapyresistant breast cancer stem cells via regulation of mitochondrial oxidative phosphorylation. Cell Metab. 26, 633-647 e637 (2017).

68. Rabindran, S. K. et al. Antitumor activity of HKI-272, an orally active, irreversible inhibitor of the HER-2 tyrosine kinase. Cancer Res. 64, 3958-3965 (2004).

69. Rusnak, D. W. et al. The effects of the novel, reversible epidermal growth factor receptor/ErbB-2 tyrosine kinase inhibitor, GW2016, on the growth of human normal and tumor-derived cell lines in vitro and in vivo. Mol. Cancer Therap. 1, 85-94 (2001).

70. Frankel, C. \& Palmieri, F. M. Lapatinib side-effect management. Clin. J. Oncol. Nurs. 14, 223-233 (2010).

71. Sodergren, S. C. et al. Systematic review of the side effects associated with anti-HER2-targeted therapies used in the treatment of breast cancer, on behalf of the EORTC quality of life group. Target. Oncol. 11, 277-292 (2016). 